\title{
Die primäre Endoprothetik bei der Versorgung von Frakturen der proximalen Tibia beim geriatrischen Patienten
}

\author{
Thomas Haufe, Peter Müller, Stefan Förch, Edgar Mayr
}

\section{Zusammenfassung}

Die Tibiakopffraktur stellt mit 1\% aller Frakturen eine seltene Verletzung dar [4]. Jedoch nimmt die Zahl der Tibiakopffrakturen im Alter stetig zu $[5,6]$. Diese entstehen, im Gegensatz zum jungen Patienten, meist durch Niederrasanztraumata. Vor dem Hintergrund der ständig an Bedeutung gewinnenden „Alterstraumatologie“ stellt die primäre Endoprothetik am Kniegelenk einen interessanten operativen Ansatz dar. Häufig handelt es sich aufgrund einer vorbestehenden Osteoporose um komplexe Frakturen mit großen Defektzonen [7]. Hierdurch wird eine konventionelle plattenosteosynthetische Versorgung erschwert und erlaubt häufig nur eine Beübungsstabilität unter Teilbelastung. Gerade die postoperative frühfunktionelle Beübung unter Vollbelastung ist aber für den geriatrischen Patienten von entscheidender Bedeutung. Die primäre Endoprothetik hingegen erlaubt postoperativ eine sofortige belastungsstabile, frühfunktionelle Beübung und kann somit dazu beitragen, sekundäre Komplikationen $\mathrm{zu}$ vermindern und die Mobilität zu erhalten.

\section{Primary Endoprosthesis in the Care of Fractures of the Proximal Tibia in Geriatric Patients}

Although proximal tibia fracture is rare and comprises only $1 \%$ of all fractures, the incidence in the elderly population is increasing. In contrast to the younger patient, for the orthogeriatric patient the underlying cause is mostly a low energy trauma. In the field of orthogeriatric traumatology, primary total knee arthroplasty seems to be a promising alternative to the open reduction and internal fixation (ORIF) of a proximal tibia fracture. As osteoporosis is common in this population, there are frequently more complex fractures with major defects in the articular surface. Because of these defects, most of the patients who undergo ORIF will only be allowed partial weight bearing postoperatively. But for the elderly patient, immediate postoperative mobilisation with full weight bearing is important. Primary arthroplasty allows immediate postoperative mobilisation with full weight bearing and may help to reduce the postoperative risks to maintaining the mobility of those patients.

\section{Einleitung}

Die elektive Knieendoprothetik bei primärer Gonarthrose ist ein fester Bestandteil im klinischen Alltag. Es handelt sich um ein etabliertes Verfahren auf Basis zahlreicher Studien. Hingegen sind Publikationen zu Erfahrungen mit der primären Endoprothetik bei komplexen Tibiakopffrakturen gering. In der aktuellen Literatur spiegelt sich dies mit ins-

OP-JOURNAL 2016; 32: 232-236

(c) Georg Thieme Verlag KG Stuttgart · New York DOI http://dx.doi.org/10.1055/s-0042-121503 gesamt wenigen Studien mit jeweils kleinen Patientenkollektiven von nicht mehr als $n=15$ Patienten wider. Die primäre Endoprothetik am Kniegelenk scheint jedoch gerade in der ständig an Bedeutung gewinnenden „Alterstraumatologie" ein interessanter operativer Ansatz zu sein. Die Tibiakopffraktur stellt mit $1 \%$ aller Frakturen eine eher seltene Verletzung dar [4].

Die Zahl der Tibiakopffrakturen im höheren Lebensalter nimmt stetig zu $[5,6]$.

Hierbei handelt es sich, im Gegensatz zum jungen Patienten, meist um Nieder- rasanztraumata. Bei 95\% der Patienten über 70 Jahren mit einer Fraktur liegt eine Osteoporose vor [14]. Hieraus resultieren häufig komplexe Plateaufrakturen in Kombination mit ausgeprägten metaphysären Substanzdefekten [15], welche die konventionelle plattenosteosynthetische Versorgung erschweren und häufig nur eine Beübungsstabilität unter Teilbelastung erlauben. Gerade die postoperative frühfunktionelle Beübung unter Vollbelastung ist aber für den geriatrischen Patienten von entscheidender Bedeutung, um die vorbestehende Mobilität erhalten zu können. Wenn aufgrund von Komorbiditäten eine Teilbelastung nicht sicher durchgeführt werden kann, erhöht sich das postoperative Risiko für eine Thrombose, Lungenembolie oder Pneumonie. Außerdem droht der Verlust von propriozeptiven Fähigkeiten und Muskelsubstanz, was zu einer gänzlichen Einbüßung der Mobilität führen kann.

\section{Indikationsstellung}

Die Indikation muss von Patient zu Patient individuell gestellt werden. Hier spielen viele verschiedene Faktoren eine Rolle.

Prinzipiell sollte über eine primär endoprothetische Versorgung immer dann nachgedacht werden, wenn eine belastungsstabile Osteosynthese nicht möglich ist und eine Teilbelastung vom Patienten nicht umgesetzt werden kann.

Kriterien hierfür sind die Frakturmorphologie, die ligamentären Begleitverletzungen und die knöcherne Situation. Zur Klassifikation der Fraktur und der ligamentären Begleitverletzungen verwenden wir die AO- und die Schatzker-Klassifikation. Hierbei empfiehlt es sich, die konventionelle Röntgendiagnostik durch eine CT zu erweitern. Bezüglich der Frakturmorphologie ist bei B-Frakturen (AO 41 B1-3) eine Versorgung mit einem ungekoppelten Prothesenmodell (z.B.e. 


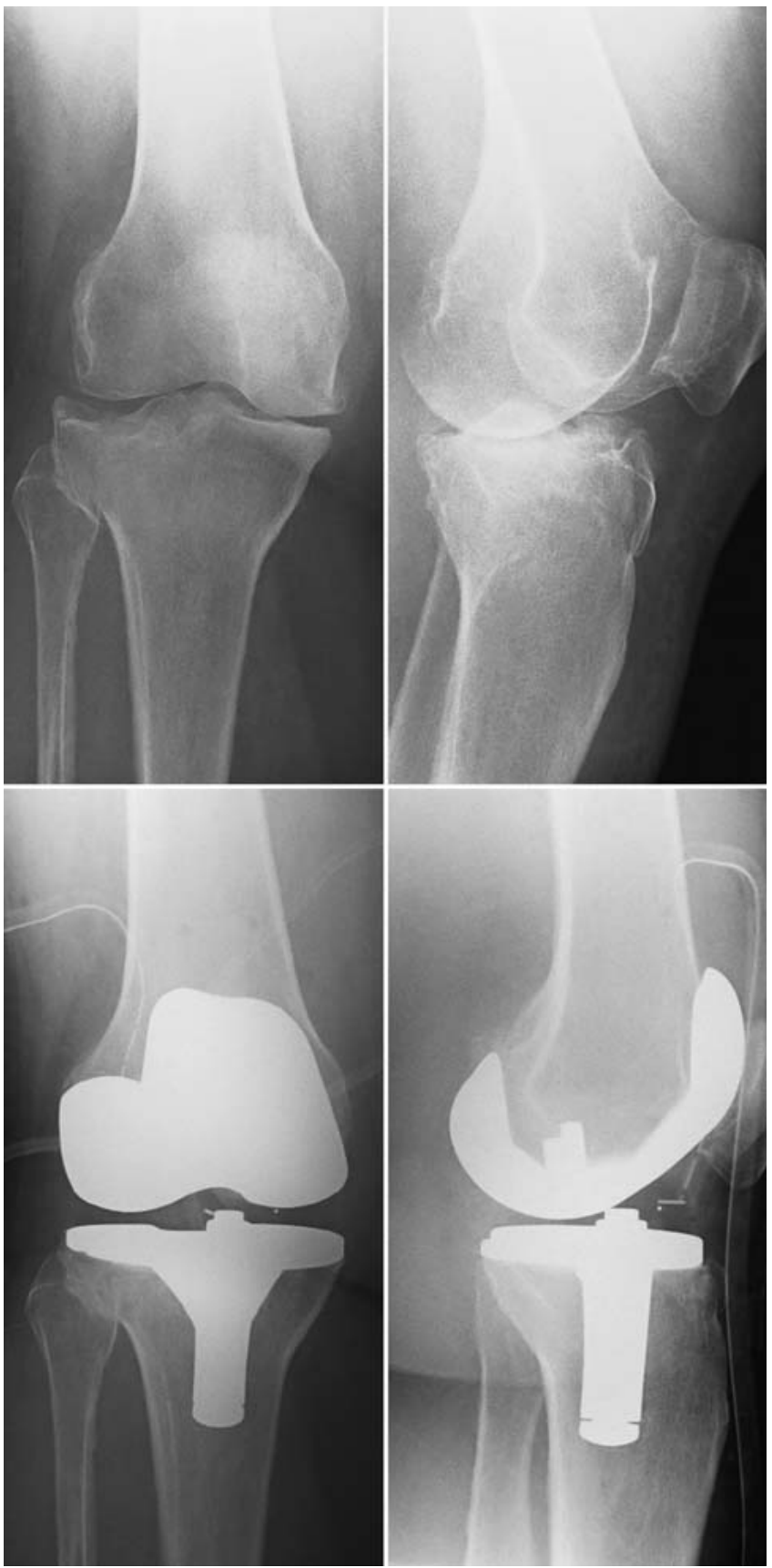

motion Knieprothese [Braun $\left.\left.{ }^{\circledR}\right]\right)$ möglich (Fallbeispiel 1, Abb. 1). C-Frakturen (AO 41 C1-3) sollten, je nach Ausmaß der ligamentären Verletzungen, mit einem Semi-constrained-System (z.B. Innex SC $\left[\right.$ Zimmer $\left.^{\circledR}\right]$ ) oder einem Full-constrainedSystem (z.B. Rotating Hinge Prothese [Zimmer ${ }^{\circledR}$ ]) versorgt werden (Fallbeispiel 2, Abb. 2). Das Vorhandensein von ligamentären Begleitverletzungen sehen wir als eine Kontraindikation für ein osteosynthetisches Verfahren. Bei der Wahl des Prothesensystems sollte hier die Schatzker-Klassifikation bedacht werden. Bei Tibiakopffrakturen, die nach Schatzker II (mit einer Impression über
Abb. 1 Weiblich, 83 Jahre, Tibiakopffraktur AO 41B3 e.motion $\left(\right.$ Braun $\left.^{\circledR}\right)$, KSS1 83, KSS2 80, WOMAC 87 (Followup 12 Monate).

\section{Operationstechnische Besonderheiten}

Wir empfehlen eine zeitnahe operative Ausversorgung binnen 12 Stunden. Sollte dies nicht möglich sein, sehen wir bei Frakturen AO C 1-3 die Indikation zur Anlage eines gelenkübergreifenden Fixateur externe. Frakturen AO B 1-3 können bei guten Weichteilverhältnissen in einer Orthese (z.B. Mecronschiene) ruhiggestellt werden. Die primäre Endoprothetik bei Tibiakopffraktur bedarf einer exakten präoperativen Planung und Vorbereitung. Das gewählte Prothesenmodell sollte in allen Größen und möglichen Erweiterungsoptionen vorrätig sein. Insbesondere bei spongiösen Defektzonen ist eine CT-Bildgebung von großer Bedeutung. Es gibt verschiedene Möglichkeiten, diese zu füllen. Hierzu bietet sich zunächst das Verwenden der Spongiosa aus den Resektionsschnitten an. Von einer Spongiosaentnahme am Beckenkamm oder Femur raten wir beim geriatrischen Patienten aufgrund der möglichen Komorbiditäten ab. Sollte die autologe Spongiosa nicht ausreichen, stellen zum einen die allogene Spongiosatransplantation und zum anderen das Verwenden von trabekulären Tantal Cones, die Press-fit verankert werden, eine Lösung dar. Diese sind vor allem aus der Revisionsendoprothetik bekannt. Des Weiteren sollte eine anatomische Reposition mit Defektanhebung nicht erzwungen werden, um den „bone stock“ der durch die Fraktur impaktierten Spongiosa nicht zu schwächen. Hier ist weniger oft mehr. Eine weitere Schwierigkeit stellen die Resektionsschnitte bei Trümmerfrakturen dar, da die Fragmente bei flektiertem Kniegelenk häufig verkippen. Dislozierte Fragmente können in diesem Fall im Plateaubereich mit isolierten Zugschrauben von dorsal oder ventral refixiert werden, um die Integrität des tibialen Gelenkblocks wiederherzustellen. Bei metaphysären Trümmerzonen ist die Verankerung mit einem Stem von entscheidender Bedeutung. Dieser sollte bis zur Mitte der Tibia reichen. Sollte auch hiermit keine ausreichende Stabilität erreicht werden können, besteht die Möglichkeit einer additiven Plattenosteosynthese (Fallbeispiel 3, Abb. 3).

\section{Grundsatzdiskussion: primäre endoprothetische Versorgung vs. Osteosynthese}

Dem Goldstandard der AO (Arbeitsgemeinschaft für Osteosynthesefragen), wonach intraartikuläre Frakturen pri- 
mär mittels ORIF (open reduction and internal fixation) versorgt werden sollten, steht das Konzept der primären Endoprothetik gegenüber. Wie erwähnt, sind Publikationen zu Erfahrungen mit der primären Endoprothetik bei komplexen Tibiakopffrakturen gering. Die wenigen Studien deuten jedoch zufriedenstellende Ergebnisse an [10-13]. Dies deckt sich mit einer von uns durchgeführten Studie, in der 22 Patienten, die mit einer primären Knieendoprothese versorgt worden waren, nachuntersucht wurden. Dieses Patientenkollektiv wurde mittels des Knee-Society-RatingSystems [8] und des WOMAC-Score-Systems [9] reevaluiert. Das durchschnittliche Follow-up waren 27 Monate mit einem Mindest-Follow-up von 12 Monaten. Alle Scores zeigten im Durchschnitt sehr gute bis gute Ergebnisse. Diese Zahlen stehen den schlechten Langzeitergebnissen nach Plattenosteosynthese bei Tibiakopffraktur beim geriatrischen Patienten gegenüber $[6,10,16,17]$. Nach Osteosynthese am Kniegelenk bei intraartikulären Frakturen wird ein 3-facher Anstieg der Arthroserate und ein Anstieg der Kondylennekroserate beschrieben [6]. Das bedingt möglicherweise spätere Folgeeingriffe. Hierbei zeigen sich jedoch bei der sekundären endoprothetischen Versorgung schlechtere Langzeitergebnisse mit erhöhter Komplikationsrate als bei der primären Endoprothetik $[2,10,16,18,19]$. Zusätzlich werden vermehrte Repositionsverluste nach osteosynthetischer Versorgung bei Tibiakopffrakturen beim geriatrischen Patienten beschrieben $[6,10,12,16,20]$. Dies ist sicherlich nur zum Teil der schlechten Knochensubstanz zuzuschreiben.

Das Erreichen einer ausreichenden Stabilität bei Impressionsspaltbrüchen und Mehrfragmentfrakturen ist mittels der konventionellen Osteosynthese häufig schwierig $[21,22]$ und in aller Regel nur teilbelastungsstabil.

Im Klinikum Augsburg haben wir eine eigene unfallchirurgische Station für geriatrische Patienten.

Die Grundprinzipien hier sind die definitive Versorgung im 1. Eingriff und eine belastungsstabile Versorgung, um eine frühfunktionelle Beübung zu ermöglichen.

Neben den eben genannten „operativtechnischen“ Problemen müssen speziell beim älteren Menschen noch „biologi-
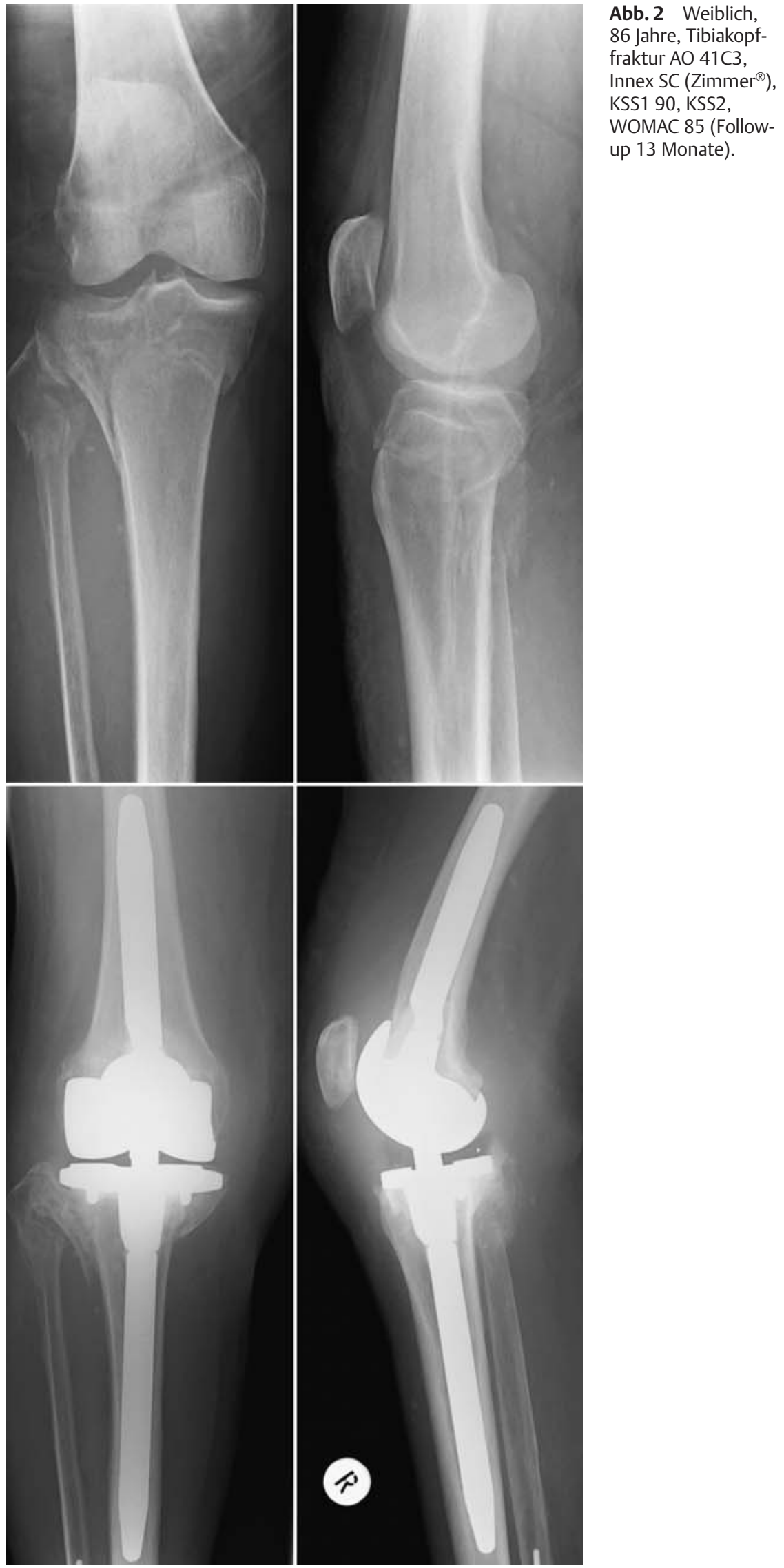

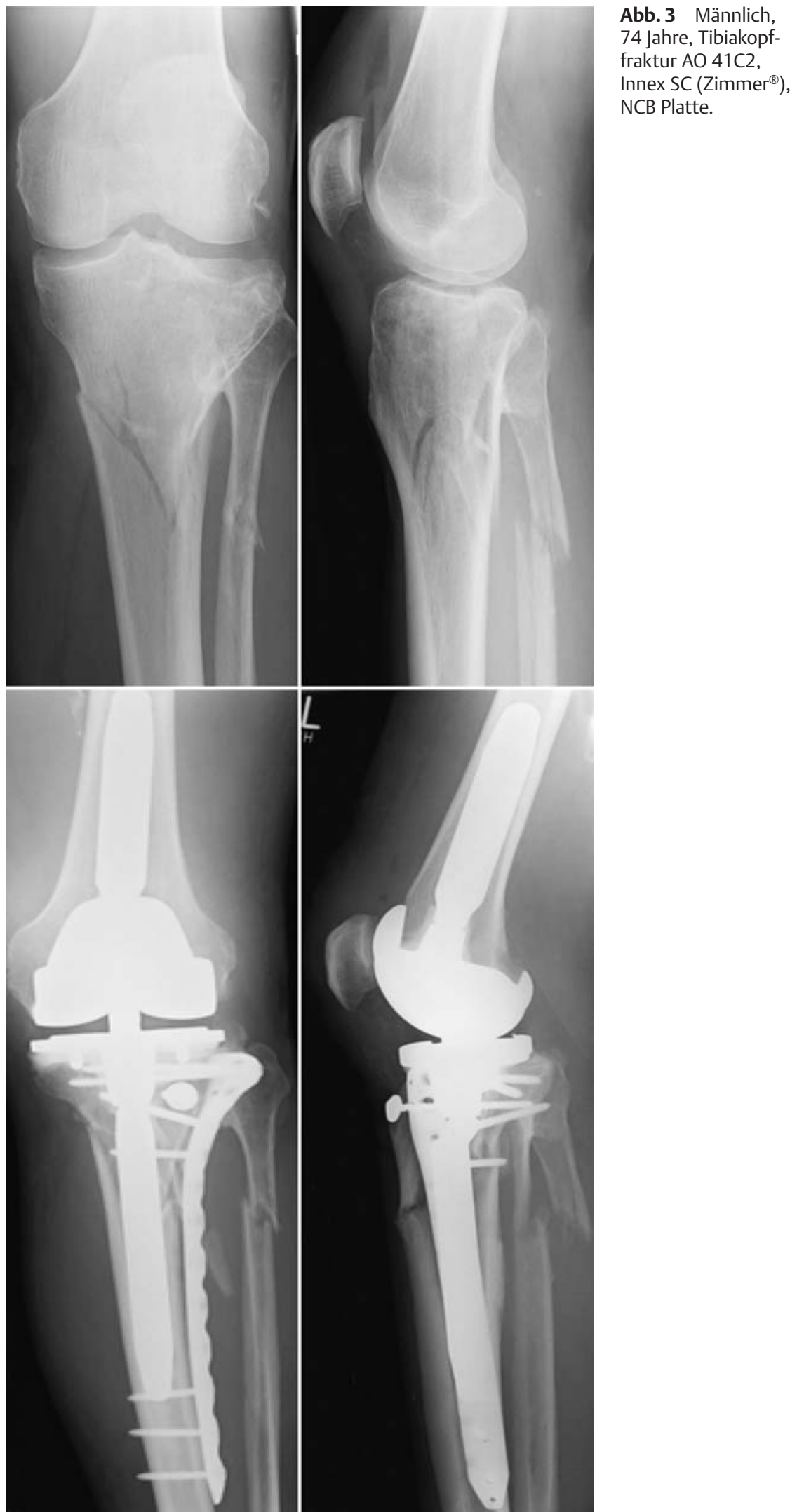

sche“ Probleme berücksichtigt werden. Aufgrund einer Sarkopenie, einer schon im Vorfeld bestehenden Einschränkung der Mobilität, kognitiven Einschränkungen/Demenz oder einem Delir ist bei die- ser Patientengruppe die Durchführung einer Teilbelastung häufig unmöglich [4]. Mit einer ab dem 1. postoperativen Tag belastungsstabilen Versorgung können Risiken wie Thrombosen, Lungen- embolien und Lungenentzündungen verringert werden. Zusätzlich bleiben propriozeptive Fähigkeiten und Muskelsubstanz erhalten. Zusätzlich können die Patienten wieder schneller an tagesstrukturierenden Maßnahmen und am Alltag teilzunehmen. Die primäre endoprothetische Versorgung in der Fraktursituation stellt den Operateur vor verschiedene technische Herausforderungen. Ein gekoppeltes Prothesenmodell ermöglicht eine ausreichende Stabilität bei oftmals schon im Vorfeld bestehenden oder durch den Unfall entstandenen ligamentären Instabilitäten. Durch den verlängerten Schaft ist eine suffiziente Verankerung im osteoporotischen Knochen möglich. Diese Vorteile wiegen das Problem der eingeschränkten Revisionsmöglichkeiten bei den verwendeten Prothesenmodellen unserer Meinung nach deutlich auf.

\section{Schlussfolgerung}

In Zeiten des demografischen Wandels stellt das Konzept der primären endoprothetischen Versorgung von Tibiakopffrakturen aufgrund seiner gezeigten Vorteile in der Alterstraumatologie eine sinnvolle Indikation zu der primär osteosynthetischen Versorgung dar. Jedoch handelt es sich hierbei nicht um einen Paradigmenwechsel oder gar ein neues Patentrezept - die primäre Endoprothetik bei Tibiakopffrakturen bedarf einer strengen Indikationsstellung und eines versierten Operateurs.

Interessenkonflikt: Nein.

\section{Literatur}

${ }^{1}$ Lohmann R, Haid K, Stöckle U et al. Epidemiologie und Perspektiven der Alterstraumatologie. Unfallchirurg 2007; 110: 553-562

2 Saleh KJ, Sherman P, Katkin P et al. Total knee arthroplasty after open reduction and internal fixation of fractures of the tibial plateau. J Bone Joint Surg Am 2001; 83-A: 1144-1148

3 Vogel T, Kampmann P, Bürklein D et al. Versorgungswirklichkeit bei osteoporosebedingten Frakturen in der deutschen Unfallchirurgie. Ein Beitrag zur Versorgungsforschung. Unfallchirurg 2008; 111: 869-877

4 Pape D, Hoffmann A, Gerich T. Frakturen des Kniegelenks im hohen Lebensalter. Orthopäde 2014; 43: 365-373

5 Bengnér U, Johnell O, Redlund-Johnell I. Increasing incidence of tibia condyle and patella fractures. Acta Orthop Scand 1986; 57: 334-336

${ }^{6}$ Gerlich T, Blauth M, Witte F et al. Kniegelenksendoprothetik nach Tibiakopffrakturen im höheren Alter. Unfallchirurg 2001; 104: 50-56

${ }^{7}$ Malviya A, Reed MR, Partington PF. Acute primary total knee arthroplasty for peri-articular knee fractures in patients over 65 years of age. Injury 2011; 42: 1368-1371 
${ }^{8}$ Insall JN, Dorr LD, Scott RD et al. Rationale of The Knee Society clinical rating system. Clin Orthop Relat Res 1989; 248: 13-14

${ }^{9}$ Bellamy N, Campbell J, Stevens J et al. Validation study of a computerized version of the Western Ontario and McMaster Universities VA3.0 Osteoarthritis Index. J Rheumatol 1997; 24: 2413-2415

${ }^{10}$ Kilian U. Kniegelenksendoprothese zur primären Therapie von intraartikulären Tibiakopffrakturen im höheren Alter. Unfallchirurg 2003; 106: 1046-1050

${ }^{11}$ Nau T, Pflegerl E, Erhardt J et al. Primary knee arthroplasty for periarticular fractures. J Arthroplasty 2003; 18: 968-971

${ }^{12}$ Nourissat G, Hoffmann E, Hémon $C$ et al. Arthroplastie totale de genou pour fracture récente grave de l'épiphyse tibiale proximale chez le sujet âgé. Rev Chir Orthop Reparatrice Appar Mot 2006; 92: 242-247

${ }^{13}$ Schwarz N, Buchinger W, Mähring $M$ et al. Knieprothese als Ersttherapie bei proximaler Tibiafraktur. Unfallchirurg 2008; 111: 928 932

${ }^{14}$ Bogoch ER, Elliot-Gibson V, Beaton DE et al. Effective initiation of osteoporosis diagnosis and treatment for patients with a fragility fracture in an orthopaedic environment. J Bone Joint Surg Am 2006; 88: 25-34
15 Petersen W, Zantop T, Raschke M. [Fracture of the tibial head]. Unfallchirurg 2006; 109: 219-232

16 Gerlich T, Bosch U, Schmidt E et al. Kniegelenkendoprothetik nach Tibiakopffrakturen mittelfristige Ergebnisse einer Kohortenanalyse. Unfallchirurg 2001; 104: 414-419

17 Ali AM, El-Shafie M, Willett KM. Failure of fixation of tibial plateau fractures. J Orthop Trauma 2002; 16: 323-329

18 Papadopoulos EC, Parvizi J, Lai CH et al. Total knee arthroplasty following prior distal femoral fracture. Knee 2002; 9: 267-274

${ }^{19}$ Weiss NG, Parvizi J, Trousdale RT et al. Total knee arthroplasty in patients with a prior fracture of the tibial plateau. J Bone Joint Surg Am 2003; 85-A: 218-221

${ }^{20}$ Honkonen SE. Indications for surgical treatment of tibial condyle fractures. Clin Orthop Relat Res 1994; 302: 199-205

${ }^{21}$ Hertel P. Tibiakopffrakturen. Unfallchirurg 1997; 100: 508-523

22 Peicha G, Grechenig W, Seibert FJ. Frühsekundäre Kniegelenksendoprothese nach Tibiakopftrümmerfrakturen. European Journal of Trauma 2001; 1: 36-39

${ }^{23}$ Vermeire J, Scheerlinck T. Early primary total knee replacement for complex proximal tibia fractures in elderly and osteoarthritic patients. Acta Orthop Belg 2010; 76: 785-793
Dr. med. Thomas Haufe

Assistenzarzt

Dr. med Peter Müller

Oberarzt

Dr. med. Stefan Förch

Oberarzt

Prof. Dr. Dr. hc. Edgar Mayr

Chefarzt

Klinik für Unfall-, Hand- und

Wiederherstellungschirurgie

Klinikum Augsburg

Stenglinstraße 2

86156 Augsburg

thomas.haufe@klinikumaugsburg.de 